\title{
A STUDY OF PRECISION IN TURNING EXTERNAL CYLINDRICAL SURFACES WITH MOVABLE TWO-BLADE BLOCK
}

\author{
Nely Georgieva ${ }^{1}$ - Pavel Petrov ${ }^{2}-$ Mihail Karshakov $^{2}$ - Zlatin Zlatev $^{1^{*}}$ \\ ${ }^{1}$ Faculty of Technics and Technologies, Trakia University of Stara Zagora, Bulgaria \\ ${ }^{2}$ Faculty of Mechanical and Manufacturing Engineering, "Angel Kanchev" University of Ruse, Bulgaria
}

\begin{tabular}{l}
\hline ARTICLE INFO \\
\hline Article history: \\
Received: 12.11 .2017$. \\
Received in revised form: 8.4 .2018$. \\
Accepted: 10.4 .2018$. \\
\hline Keywords: \\
Movable two-blade block \\
Axis displacement inserts \\
Variable allowance \\
Adjustment elastic deformation of a cutting block \\
\hline DOI: http://doi.org/10.30765/er.39.2.10
\end{tabular}

\section{Introduction}

Processing of cylindrical surfaces with high quality requirements continues to be one of the ongoing problems of the modern engineering. In the present state, there is multiple research in the area of the precision treatment of these surfaces [1-3]. They will

\begin{abstract}
:
In practice, movable blocks with oppositely arranged cutting inserts are used for turning internal and external cylindrical surfaces with great length. With these instruments, the dimension of dynamic adjustment is formed as a result of the continuous striving to the dynamic equilibrium of the radial components of both operation cutting forces which is restricted in the systems of the tool and the work piece. In this processing scheme, it is particularly important that the opposite cutting edges lie in a plane perpendicular to the axis of the work piece, which is difficult for implementation in certain circumstances. In addition, under certain circumstances, the variation of the machining allowance has an impact on the size of the dynamic adjustment. It leads to reduction of the diameter of the treated surface towards the dimension of the static adjustment of the block.

This article proposes an innovative solution for using tools with opposite cutting inserts, which are axially displaced with guaranteed value to increase the accuracy of the obtained size. The question of adjusting the elasticity of the cutting block to compensate for the effect of the variable allowance on the accuracy of the treatment is investigated. As the result of researching, the dependencies to quantify from this impact and the conditions for its reduction are received, which can be used in practical conditions for increasing accuracy of the static adjustment.
\end{abstract}

have positive effect when they are used in specific circumstances. For the details with great length of their surfaces, these are particularly important methods and tools to increase the productivity of their production. This has a markedly positive effect. Once they are used in practice decisions, mainly for internal cylindrical surfaces, they are being processed

\footnotetext{
* Corresponding author

E-mail address: zlatin.zlatev@trakia-uni.bg
} 
through a combined tool with skiving and burnishing (by surface plastic deformation) parts [4-6]. This vastly decrease the time for treatment. The main manufacturer of such tools is ECOROLL AG, Germany. Their team has many registered patents where the find information could be found on specific design solutions under different handling conditions of external and internal cylindrical surfaces.

[7] suggests such kind of a tool with one insert. The main disadvantage of this decision is the missing possibilities to be equilibrated by the radial cutting forces. When skiving part is as self-established movable two-blade block (MTBB) with oppositely disposed cutting inserts this problem is solved [8-9], and greater stability of the tool is achieved. This technical solution provides achieving a high precision of the diametrical size over the entire length of the surface. This approach has limited use for external cylindrical surfaces and one of the reasons for that is its insufficient research.

When working with the MTBB with opposite location of the cutting inserts, the size of dynamic adjustment is formed as a result of the continuous striving to the dynamic equilibrium radial components of both inserts in the systems of the tool and the work piece. To ensure precision of treatment certain circumstances are needed to be provided, most important of which are: the same name opposite points of the main cutting edges must lie in planes perpendicular to the axis of rotation; the MTBB stability in the radial direction must be maximum etc. r. In practice, the first prerequisite is difficult to be realized, and one of the main cutting edges of the two cutting inserts is frequently included earlier in the cutting process, ahead of the other with the dimension of their displacement in the axial direction. This leads to a reduction of the diameter of the treated surface towards the dimension of the static adjustment of the block [10-11] and compromises the accuracy of the size obtained.

In [12] a research has been performed of the influence of axial displacement of the inserts on combined processing by the movable block. In [13-14] the impact of the elasticity of the instrument system was examined on the accuracy of the obtained size.

On the basis of these studies, the research was done between the relationship of the axial displacement of the cutting inserts and the accuracy achieved in adjusting the elasticity of the cutting block. This was done in the presence of a variable processing allowance with the aim to receive practical dependencies for static adjustment of the tools.

\section{Statement}

The movable two-blade block is adjusted to the size in advance and the dimension of the static adjustment is not corrected during the working cycle. Therefore, in the batch processing of the parts, the relationship between the tolerance of the work piece diameter and that of the treated surface depends on the stability of the technological system. In this case it only includes the work piece and the MTBB.

The object of the researching is the assessment of the impact of the displacement of the opposite inserts in the axial direction on the above mention relation, i.e. over the accuracy of the cutting stroke. To relieve the research. next preconditions can be introduced:

$\checkmark \quad$ in the absence of axial displacement of the cutting inserts the plane containing their tops is perpendicular to the axis of rotation, and the axes of symmetry of the block and the work piece coincide with the last;

$\checkmark$ tool minor cutting edge angles $\kappa_{\mathrm{r}}$ are zero degrees and tool cutting edge angles $\kappa_{\mathrm{r}}$ are equal;

$\checkmark$ the deviations of the surface form of the work piece is insignificant;

$\checkmark$ the friction in the guides of the block is overlooked;

$\checkmark$ primary rotational motion is carried out by the work piece, the feed motion - by the tool with a speed $f, \mathrm{~mm} / \mathrm{rev}$

$\checkmark \quad$ the bodies of the MTBB and the work piece are non-deformable.

The displacement $(X)$ of the inserts leads to a reduction of the diameter of the treated surface toward the dimension of the static adjustment which is a result of radial displacement of the MTBB to the outstripping insert and is characterized by a limit value $\left(X_{1}\right)$, after which the radial displacement receives constant maximum value [9]. The reason for this is the selfestablishment of the MTBB for satisfaction of the dynamic equilibrium. When reducing the tool cutting edge angle $\kappa_{\mathrm{r}}$ in the range of $90^{\circ}-0^{\circ}$ the value of the limit axial displacement increases starting respectively from $f / 2$. In case of axial displacement less than the limit $X<X_{1}$ there is a mutual influence of the cutting inserts, which is leaded to difference in the shape and dimensions of the sheared from them layers.

Figure 1 schematically shows the processing of a shaft having axially displaced cutting inserts, of which the bottom one is outstripped and its area of 
shear layer is hatched and denoted by $A_{11}$, respectively, of the lagged - $A_{21}$. From condition of the radial cutting forces equilibrium the two areas are equal to each other $\left(A_{11}=A_{21}\right)$, and as a result of their axial displacement $X_{1}$ also equality of their technological dimensions exists - cutting depth $a_{\mathrm{p} 1}=a_{\mathrm{p} 2}$ and feed $f$. The magnitude of the limit axial displacement is determined by dependence (1).

$$
X_{1}=\frac{f}{2}+\frac{d_{\mathrm{w}}-L_{\mathrm{a}}}{3 \operatorname{tg} \kappa_{\mathrm{r}}}
$$

where $d_{\mathrm{w}}$ is diameter of the work piece; $L_{\mathrm{a}}$ - dimension of the static adjustment of the MTBB, which is generally the distance between the trajectories of the tips of the cutting inserts in direction of the feed movement (increasing axial displacement over the value obtained in (1) does not change the shape and dimensions of hatched sections).

The other designations in Figure 1 have the following meaning:

$A_{10}$ и $A_{20}$ - areas of the sheared layers respectively from the first and second inserts with absent axial displacement between them;

$a_{\mathrm{p}}-$ cutting depth without axial displacement of the cutting inserts;

$\Delta_{\mathrm{r}}$ - radial displacement of the block as a result of the axial displacement of the inserts from the condition of equilibrium of the radial cutting forces;

$d_{1-}$ diameter received by outstripping cutting insert;

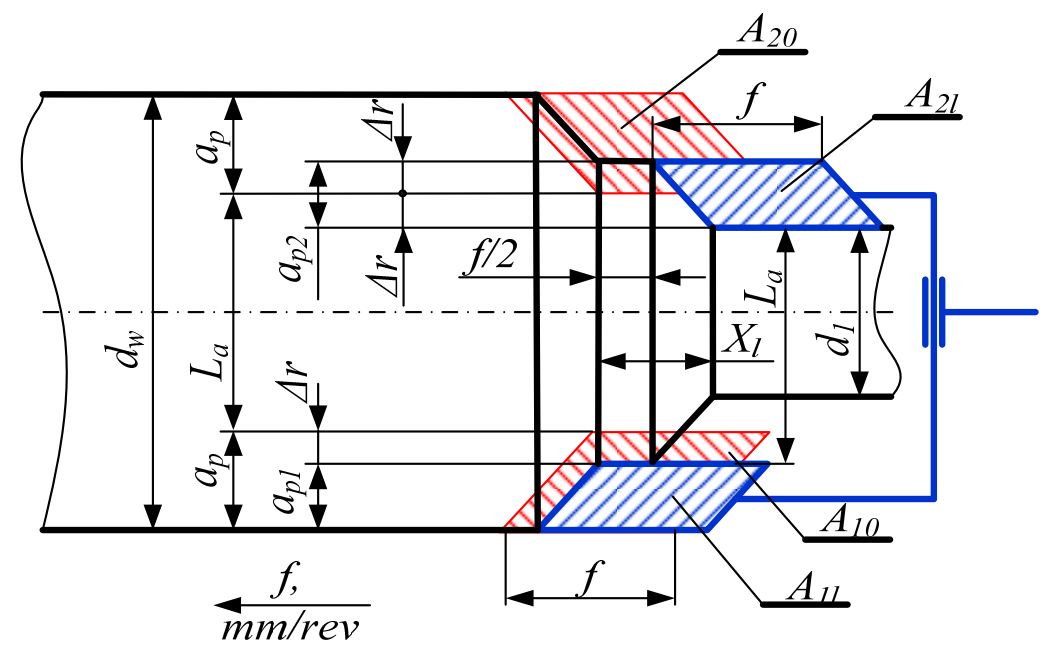

Figure 1. Scheme of turning the MTBB with axially displaced inserts.

$d$-diameter of the treated surface without axial displacement of the cutting inserts;

$d_{1}$ - diameter of the treated surface, as a result of the limit axial displacement.

From the analysis of the figure and the above mention conditions, it can be established that at zero axial displacement $(X=0)$ a diameter of the treated surface, equal to the static adjustment will be formed $\left(L_{\mathrm{a}}=d\right)$ and the two inserts will operate at the same cutting depths (2).

$$
a_{\mathrm{p}}=\frac{d_{\mathrm{w}}-L_{\mathrm{a}}}{2}
$$

In case of the axial displacement $X_{1}$ both equal depths of cut will have value (3).

$$
a_{\mathrm{p} 1}=a_{\mathrm{p} 2}=\frac{d_{\mathrm{w}}-L_{\mathrm{a}}}{3}
$$

and the diameter of the treated surface is (4):

$$
d_{1}=L_{\mathrm{a}}-\frac{d_{\mathrm{w}}-L_{\mathrm{a}}}{3}=\frac{4 L_{\mathrm{a}}-d_{\mathrm{w}}}{3}
$$

The radial displacement of the MTBB is towards the first insert. In this case it gets maximum value, determined by the dependence (5):

$$
\Delta r=\frac{d_{\mathrm{w}}-L_{\mathrm{a}}}{6}
$$

Under these conditions and work of the MTBB it is necessary to establish the fluctuation of the allowance for processing affects on the diameter of the treated surface. For this purpose, the pattern of cutting (in Figure 1) is viewed in cross-section and is shown in Figure 2. There 1 denotes the outstripping 
insert (blade), and 2 - lagging and the following additional designations are introduced: $\Delta Z$ - unilateral increasing of the allowance; $d_{\mathrm{w}}^{l}, d_{\mathrm{w}}^{u}-$ upper and lower limit sizes of the work piece; $d_{1}^{l}, d_{1}^{u}$ - upper and lower limit sizes of the treated surface; $a_{\mathrm{p} 1}, a_{\mathrm{p} 2}$ - cutting depth at the lower limit sizes of the work piece; $a_{\mathrm{p} 1}, a_{\mathrm{p} 2}$ - cutting depth at the upper limit sizes of the work piece.

In Figure 2, as a starting case processing of the work piece with size $d_{w}^{l}$ is accepted. Since in both cases the size of the static adjustment is the same and it must meet condition (3), when processing work piece with size $d_{\mathrm{w}}^{u}=d_{\mathrm{w}}^{l}+2 \Delta Z$ the MTBB is self-established. The additional radial displacement $\Delta$ in the direction of the blade 1 is performed. For the determination of $\Delta$ it is convenient to use the next laying (6):

$$
\begin{aligned}
& a_{\mathrm{p} 1}=a_{\mathrm{p} 2}=a_{\mathrm{p}}, \\
& a_{\mathrm{p} 1}^{\prime}=a_{\mathrm{p} 2}=a_{\mathrm{p}},
\end{aligned}
$$

whereby, under Fig. 2a, in a force is the equality (7):

$$
a_{\mathrm{p}}+\Delta Z-\Delta=a_{\mathrm{p}}+2 \Delta
$$

and after conversion (8):

$$
\Delta=\frac{\Delta Z}{3} .
$$

From a technological point:

$$
\Delta Z=\frac{d_{\mathrm{w}}^{u}-d_{\mathrm{w}}^{l}}{2}=\frac{T d_{\mathrm{w}}}{2}
$$

where $T d_{\mathrm{w}}$ is the tolerance of the diameter of the work piece.

After substituting of (9) in (8):

$$
\Delta=\frac{T d_{\mathrm{w}}}{6}
$$

The tolerance of the diameter of the treated surface is (11):

$$
T d_{1}=d_{1}^{u}-d_{1}^{l},
$$

where $d_{1}^{l}=d_{1}^{u}-2 \Delta$.
After substituting of (10) and of (11):

$$
T d_{1}=\frac{T d_{\mathrm{w}}}{3}
$$

Correlation (12) indicates that with the maximum stability of the MTBB and axial displacement of the cutting inserts, equal to or greater than the boundary, the field of dissipation of the tolerance of the processing work pieces of a batch can be reduced theoretically 3 times.

If the premise of resistance to mechanical deformation of the body of the MTBB is dropped down to minimum, respectively maximum, allowance will cause small $\varepsilon_{1}$ or large $\varepsilon_{\mathrm{u}}$ deformations of the body of the block, which will increase the size of the static adjustment $L_{\mathrm{a}}$. Moreover, according to formula (4), the limit size of the treated surface is $(13,14)$ :

$$
\begin{aligned}
& d_{1}^{u}=\frac{4\left(L_{\mathrm{a}}+\varepsilon_{1}\right)-d_{\mathrm{w}}^{l}}{3}, \\
& d_{1}^{l}=\frac{4\left(L_{\mathrm{a}}+\varepsilon_{\mathrm{u}}\right)-d_{\mathrm{w}}^{u}}{3},
\end{aligned}
$$

and then replacing (13) and (14) into (11) and the conversion is obtained:

$$
T d_{1}=\frac{T d_{\mathrm{w}}}{3}-\frac{4}{3}\left(\varepsilon_{\mathrm{u}}-\varepsilon_{1}\right)
$$

It is obviously the existence of the theoretical possibility $T d_{1}=0$ that if the increase of elastic deformation when passing from minimum to maximum allowance satisfies the condition (16):

$$
\Delta \varepsilon=\varepsilon_{\mathrm{u}}-\varepsilon_{1}=\frac{T d_{\mathrm{w}}}{4}=\frac{\Delta Z}{2}
$$

This case is illustrated graphically in Figure 2b, which is analogous to Figure 2a designations and the output dimension of the work piece. In case of building a mutual location of the cutting inserts (blades) 1 and 2 and the work piece as a result of the increase in allowance of $\Delta \mathrm{Z}$, it is taken into account that provoked from this increase of the radial displacement $\Delta$ of the MTBB (Figure 2a) is offset by accompanying elastic deformation $\Delta \varepsilon$ which is satisfying the condition (16). Withal, the second (size forming) 
a blade keeps radial position relative to the axis of the treated surface, i.e. its diameter $d_{1}$ is not changed.

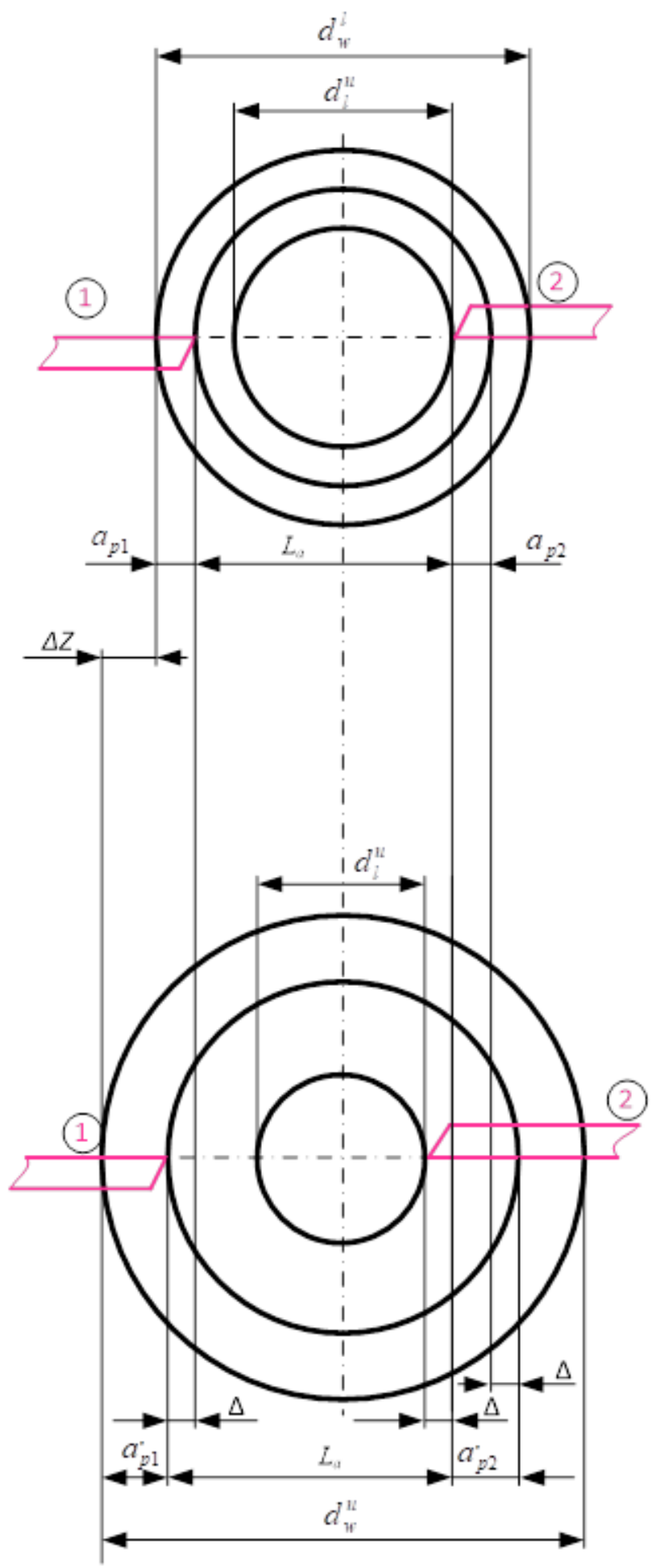

a)
Moreover, the size of dynamic adjustment $L_{\mathrm{d}}$ is formed between the blades and the conditions (6) in order for equal cutting depth to be satisfied.

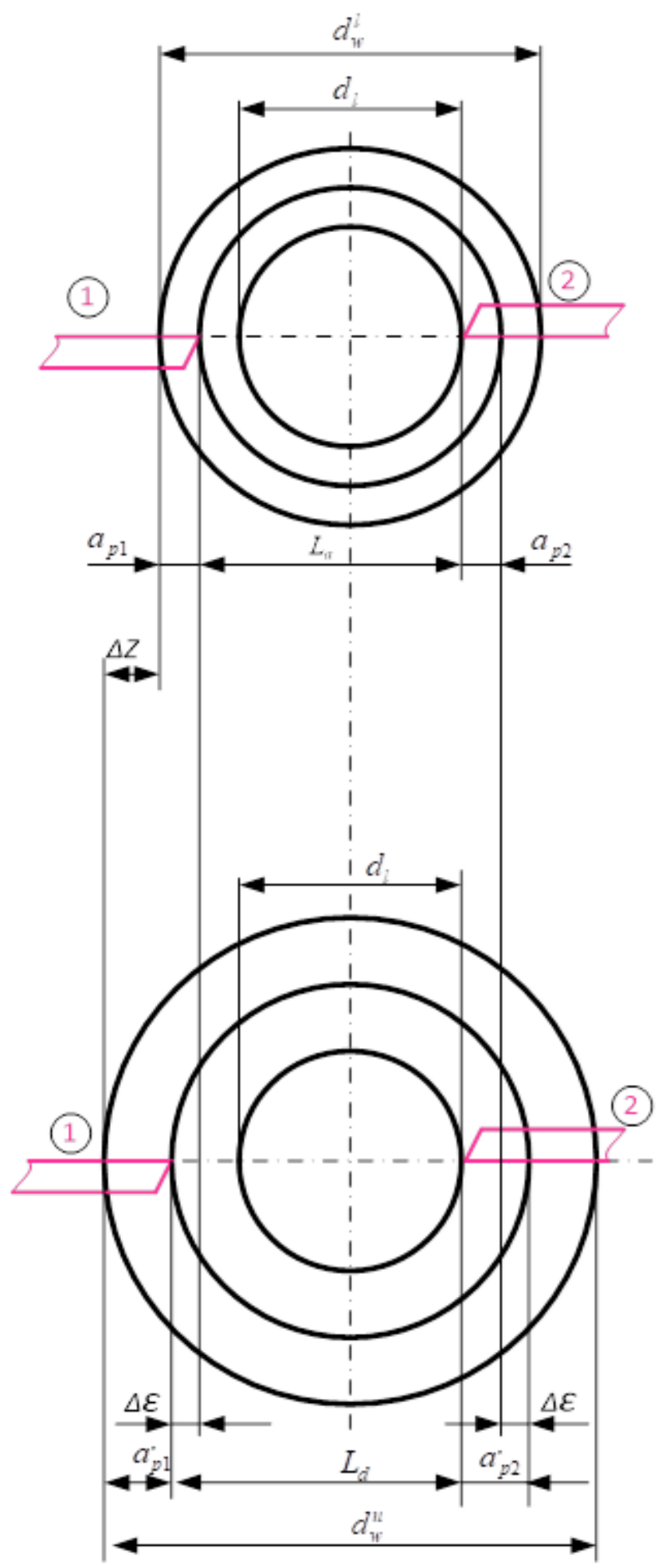

b)

Figure 2. Impact of allowances fluctuations on the diameter of the treated surface: a) with a non-deformable block b) with a deformable block. 
From the analysis of the figure, the equality can be compiled (17):

$$
a_{\mathrm{p} 1}+\Delta Z-\Delta \varepsilon=a_{\mathrm{p} 2}+\Delta \varepsilon
$$

where, after conversion, dependence (16) is obtained. It follows from this that the relevant inequalities are in force (18):

$$
\begin{aligned}
& 0 \leq \Delta \varepsilon \leq \frac{T d_{\mathrm{w}}}{4} \\
& \frac{T d_{\mathrm{w}}}{3} \geq T d_{1} \geq 0
\end{aligned}
$$

Based on the above circumstances, it can be concluded that the size of static adjustment for the MTBB with maximum stability (adopted as a nondeformable) and with regulated stability (adopted as deformable) according to dependencies (15) and (16) will be different. In both cases it must provide a diametric size near the lower limit of the tolerance field, whereat with a non-deformable block, according to (4):

$$
L_{\mathrm{a}}=\frac{3 d_{1}^{l}+d_{\mathrm{w}}^{u}}{4},
$$

and with a deformable block, according to (14):

$$
L_{\mathrm{a}}=\frac{3 d_{1}^{l}-4 \varepsilon_{\mathrm{u}}+d_{\mathrm{w}}^{u}}{4}
$$

In case of a known elastic characteristic of the MTBB their deformations can be determined by dependencies (22):

$$
\varepsilon_{1}=\frac{F_{\mathrm{P}}^{\min } . \Delta \varepsilon}{\Delta F_{\mathrm{P}}} \quad \text { and } \quad \varepsilon_{\mathrm{u}}=\frac{F_{\mathrm{P}}^{\max } . \Delta \varepsilon}{\Delta F_{\mathrm{P}}}
$$

where $\Delta F_{\mathrm{P}}=F_{\mathrm{P}}^{\max }-F_{\mathrm{P}}^{\min }, N$.

Based on (15), figure 3 shows a graphic interpretation in which the tolerance of the diameter after turning $T d_{1}$ and the elastic deformation $\Delta \varepsilon$, characterizes the stability of the MTBB and is expressed by tolerance of the diameter of the work piece $T d_{\mathrm{w}}$.

This allows for both qualitative and quantitative assessment of dependence (15), which is an equation of a straight line. In the case of Figure 3 it is built under the variation $\Delta \varepsilon$ in range from 0 to $T d_{\mathrm{w}} / 2$, at which $T d_{1}$ receives the constant value $T d_{\mathrm{w}} / 3$. In fig. 3 the straight line is depicted only for the positive values because the tolerance $T d_{1}$ cannot be negative.

In Figure 3 it can be seen, that for non-deformable block the MTBB $(\varepsilon=0)$ with the axial displacement of the cutting inserts $\left(X \geq X_{1}\right)$ the maximum theoretical possibility to reduce the field of dispersion of the diameter of the work piece is expressed by the dependence $T d_{1}=T d_{\mathrm{w}} / 3$. With reduction in stability of the MTBB the difference $\Delta \varepsilon$ between elastic deformations caused by fluctuations in the diameter of the work piece within the field of distraction $T d_{\mathrm{w}}$ increases, whereupon the above-mentioned opportunity it grows. If the elastic characteristics of the body of the MTBBis set constructively so that turning of the work piece with upper $d_{\mathrm{w}}^{u}$ and lower $d_{\mathrm{w}}^{l}$ limit sizes to cause differences in elastic its deformations $\Delta \varepsilon$ with dimension $T d_{\mathrm{w}} / 4$, then the received diameters will be the same, i.e. the field dissipation of these dimensions will be $T d_{1}=0$. Achieving the elastic characteristics of the body of the MTBB below the satisfying above condition, it leads to increased margins dispersion of diameter after turning. In case of the elastic characteristics providing $\Delta \varepsilon=T d_{\mathrm{w}} / 2$ the capabilities of deformable MTBB is aligned to that of the non-deformable block, i.e. $T d_{1}=T d_{\mathrm{w}} / 3$.

Obviously, when turning with the cutting inserts, axially displacing at a distance of $X \geq X_{1}$ the presence of the elastic deformations of the MTBB leads to the increasing of its precision capabilities. Moreover. the dependency is not synonymous - with reducing of the stability of the MTBB these opportunities reach its maximum in which $T d_{1}=0$, then begin to decline. The limit values for chosen range of amending stability in Figure 3 correspond to the one and the same values of $T d_{1}$, respectively for the non-deformable and deformable MTBB - expanding this range is pointless by technological considerations.

Of course, that actual results could differ from theoretical predictions, the cause of which can be uncertainty in measurement [15-16] and dependencies for the cutting forces mainly by inhomogeneity of the material of the work piece and wear of the cutting inserts This would result in a change of a difference $\Delta F_{\mathrm{P}}$ between cutting forces at the minimum and maximum allowances. Then, in a given elastic characteristic of the MTBB (23):

$$
\Delta \varepsilon=\frac{\Delta F_{\mathrm{p}}}{\operatorname{tg} \alpha}
$$


where $\alpha$ is the angle of inclination of the elastic characteristic $F_{\mathrm{p}}=f(\varepsilon)$ (the change will require a value of $\Delta F_{\mathrm{P}}$, other than under condition (15)).

The impact of the scattering of $\Delta \varepsilon$ according to Figure 3 on the precision opportunities of the MTBB can be seen from Table 1 .

It should be noted that the tendency for change of the $\Delta \varepsilon$ is pronounced because theoretical value is determined only by $T d_{\mathrm{w}}$. Its dependence on $\Delta F_{\mathrm{P}}$ due to the above mentioned reasons is less likely to be realized as they have equal influence on both the minimum and maximum cutting forces, wherein the difference between them changing is relatively little. The resulting dependencies and conclusions have been applied in practice and their working ability has been demonstrated by designing a tool with adjustable features of the movable two-blade block in [17] and [18].

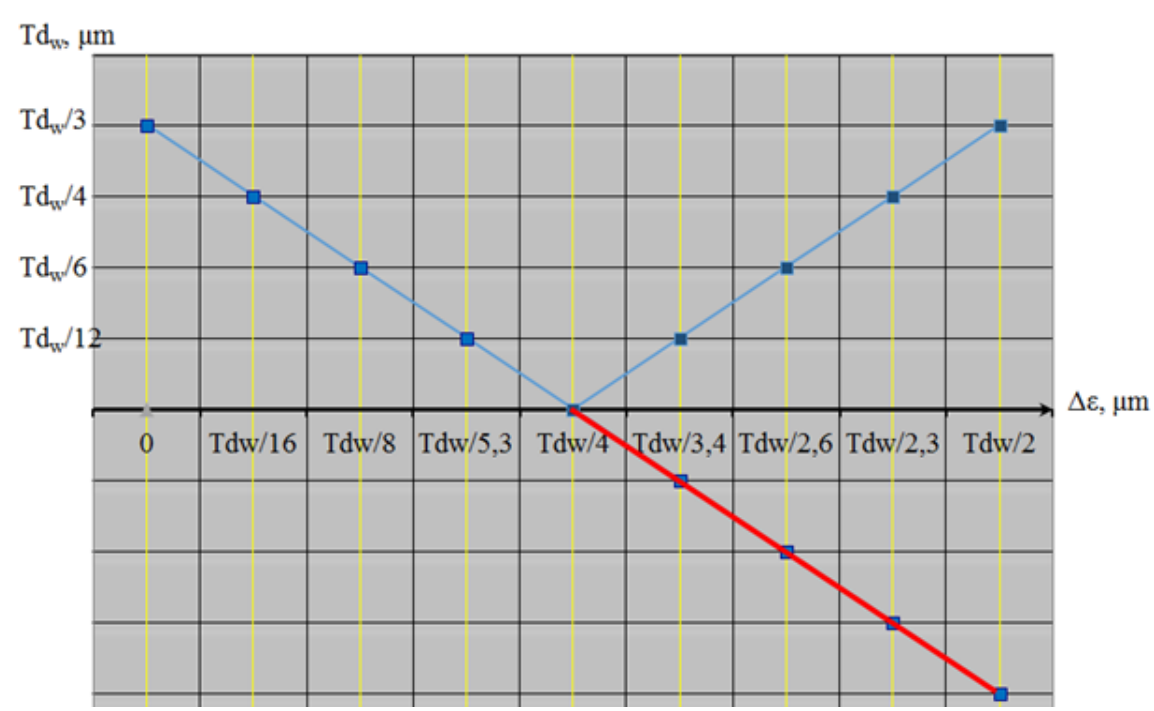

Figure 3. Dependence between the diameter tolerance after turning $T d_{l}$ and the stability (elasticity) $\Delta \varepsilon$ of the MTBB with the axially displaced inserts at a distance of $X \geq X_{1}$ expressed by the diameter tolerance of the work piece $T d_{\mathrm{w}}$.

Table.1. Effect of the scattering of $\triangle \varepsilon$ on the precision capabilities of the MTBB

\begin{tabular}{|c|c|c|}
\hline \multicolumn{2}{|c|}{ Dispersion of the $\Delta \varepsilon$} & \multirow[b]{2}{*}{$\begin{array}{l}\text { Minimum decrease of the } T d_{\mathrm{w}} \text {, corresponding to values of the } \\
\text { limits of the range, } \%\end{array}$} \\
\hline Expressed through $T d_{\mathrm{w}}$ & $\begin{array}{c}\text { Expressed through } \\
\qquad T d_{\mathrm{w}}, \%\end{array}$ & \\
\hline$T d_{\mathrm{w}} / 4$ & 0 & 100 \\
\hline$T d_{\mathrm{w}} / 5.33: T d_{\mathrm{w}} / 3.2$ & 12.5 & 91.66 \\
\hline$T d_{\mathrm{w}} / 8: T d_{\mathrm{w}} / 2.66$ & 25 & 83.33 \\
\hline$T d_{\mathrm{w}} / 16: T d_{\mathrm{w}} / 2.28$ & 37.5 & 75 \\
\hline $0: T d_{\mathrm{w}} / 2$ & 50 & 66.66 \\
\hline
\end{tabular}

\section{Conclusion}

As a result of the study the following conclusions can be drawn:

1. It's proved that processing the outer surface by the MTBB with guarantee axial displacement of the cutting inserts can lead to increasing accuracy of the turning.
2. It's proved that processing the outer surface by the MTBB with the axial displacement of the cutting inserts (blades) equal to or greater than the limit, results in a reduction of their loading. This is because, according to the relation (3) higher efficiency of turning is achieved, which is expressed in the withdrawal of greater allowance at smaller cutting depths.

3 . The newest information found in this paper is that at the MTBB with the mentioned axial displacement 
of the inserts, in contrast to the known comprehension, enhancing the block body stability leads to a reduction of its precision features. Then upon reaching the non-deformability, decreases to thrice the field of dispersion of the treated surface relative to the work piece (12) and with decreasing of the stability these options are increased (15).

4. It has been shown that there are certain values of the elastic deformation of the MTBB with axial displacement inserts, equal to or greater than the limit (16), at which the increase of diameter of the work piece does not lead to a change of the diameter of the treated surface.

5. As a result of the conducted study incurred findings make it possible to significantly improve the accuracy of turning external surfaces using the MTBB with axially displaced inserts. On the base of these results, a new construction is designed and tested in practical conditions of a tool, which has elastic characteristics satisfying the condition (15) and reach maximum accuracy.

\section{References}

[1] Cukor, G., Jurković, Z.: Optimization of turning using evolutionary algorithms, Engineering Review, 30-2 (2010) 1-10.

[2] Kundrák, J., Gyáni, K., Deszpoth, I., Sztankovics, I.: Some topics in process planning of rotational turning, Engineering Review, 34-1 (2014) 2332.

[3] Krolczyk, G., Maruda, R., Nieslony, P., Wieczorowski, M.: Surface morphology analysis of Duplex Stainless Steel (DSS) in Clean Production using the Power Spectral Density, Measurement, 94 (2016) 464-470.

[4] Dickinson, L.: US 4367576 - Skiving and roller burnishing tool, (1983).

[5] Seume, K.: DE 19934393 - Kombinertes Werkzeug zur Bear bietung von rotatiossymmetrschen Bohungen in Werkstücken, (2001).

[6] Löschner, R., Bastian, S.: EP 1512492B1 - Skiving and rollburnishing tool, (2007).

[7] Maiß, O., Denkena B., Grove T., Hybrid machining of roller bearing inner rings by hard turning and deep rolling, Journal of Materials Processing Technology, 230 (2016) 211-216.

[8] Karshakov, M.: Researching the behaviour of movable two-cutter blocks in case of skiving apertures, Mechanika-Technologija, Kaunas, (2009) 62-62.
[9] Karshakov, M.: Theoretical-experimental development of the basics of the combined treatment of deep holes by cutting and surface plastic deformation, Habilitation Work, Ruse, (2009). (in Bulgarian)

[10] Grigorov, V., Georgieva, N., Karshakov, M., Dimitrov, D.: Investigation of the size formation of Turning Shafts with a movable two-blade block, Machine building and Machine Science, 7-3 (2012) 41-47. (in Bulgarian)

[11] Zhang, X., Huanga, R., Liu, K., Kumar, A., Shan, X.: Rotating-tool diamond turning of Fresnel lenses on a roller mold for manufacturing of functional optical film, Precision Engineering, 51 (2018) 445-457.

[12] Kostadinov, V.: Investigation of the influence of axial displacement of the blades on combined processing by movable block, Proceedings of VTU Angel Kunchev Ruse, 32 (1989). (in Bulgarian)

[13] Grigorov, V., Kostadinov, S., Petrov, P.: On the determination of the dynamic characteristics of a movable block in accordance with its elastic properties, Journal of Manufacturing and Machine Knowledge, 4-1 (2008) 39-42. (in Russian)

[14] Karshakov, M., Grigorov, V., Kostadinov, S.: Influence of rubbing by sliding of a behavior of a floating cutting block with sizable elastic features. Mechanika-Technologija, Kaunas, (2009) 158-162.

[15] Sakakushev, B., Georgiev, G., Zhelezarov, I.: The first steps to construct an uncertainty budget for photogrammetric measurements in engineering, Information Processing Systems, 2 (2015) 54-57. (in Russian)

[16] Nieslony, P., Krolczyk, G., Wojciechowski, S., Chudy R., Zak, K., Maruda, R.: Surface quality and topographic inspection of variable compliance part after precise turning, Applied Surface Science, 434 (2018) 91-101.

[17] Petrov, P., Georgieva, N., Karshakov, M.: Study of precision capabilities of movable two-blade skiving block with inserts shifted on the axis, Applied Research on Technics, Technologies and Education, 4-3 (2016) 191-196.

[18] Petrov, P., Georgieva, N., Karshakov, M.: Combined tool with possibilities for one-time machining of opening of hydraulic cylinders, Applied Research on Technics, Technologies and Education, 4-3 (2016) 197-20 